quatrième série-tome 46 fascicule 5 septembre-octobre 2013

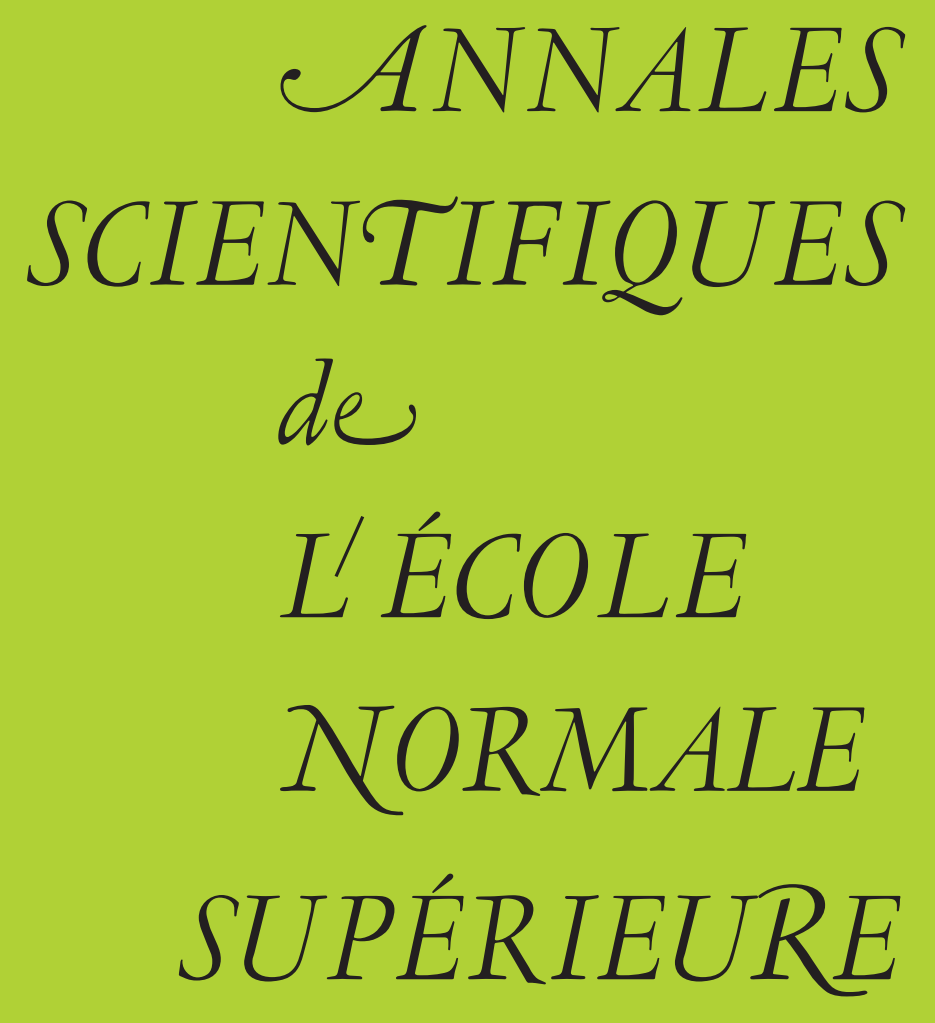

Chloé PERIN

Erratum to:

Elementary embeddings in torsion-free byperbolic groups 
Ann. Scient. Éc. Norm. Sup.

$4^{\text {e }}$ série, t. 46,2013 , p. 851 à 856

\title{
ERRATUM TO: ELEMENTARY EMBEDDINGS IN TORSION-FREE HYPERBOLIC GROUPS
}

\author{
BY CHLOÉ PERIN
}

The notations adopted are those of [1].

Proposition 5.11 of [1] states that if a torsion-free hyperbolic group $A$ admits a cyclic JSJ-like decomposition $\Lambda$, and a non injective morphism $f: A \rightarrow A$ which restricts to conjugation on each non surface type vertex group, and sends surface type vertex groups to non abelian images, then there is a retraction $r: A \rightarrow A^{\prime}$ which gives $A$ a structure of hyperbolic floor over $A^{\prime}$.

Unfortunately, we realised that Proposition 5.11 fails to hold in a few exceptional low complexity cases. The natural modification to overcome this mistake is to proceed to a slight generalization of the notion of hyperbolic floors and hyperbolic towers, which we present in Section 1.

As we will see in Section 2, however, this does not affect Theorem 1.2, the main result of the paper. Moreover, Theorem 1.2 is the only result which directly uses Proposition 5.11 in its proof.

For a corrected version of the paper, see [2]. We sincerely apologize for any confusion caused by this mistake.

\section{Modification of Proposition 5.11: extended hyperbolic towers}

To be made correct, the statement of 5.11 needs to be slightly weakened to

Proposition 1. - Let $A$ be a torsion-free hyperbolic group. Let $\Lambda$ be a cyclic JSJ-like decomposition of $A$ which does not consist of a single surface type vertex.

Assume that there exists a non injective preretraction $A \rightarrow A$ with respect to $\Lambda$. Then there exists a subgroup $A^{\prime}$ of $A$ and a retraction $r: A \rightarrow A^{\prime}$ such that $\left(A, A^{\prime}, r\right)$ is an extended hyperbolic floor. Moreover given a non surface type vertex group $R_{0}$ of $\Lambda$ we can choose $A^{\prime}$ to contain $R_{0}$.

where extended hyperbolic floors are given by 
Definition 2 (extended hyperbolic floor). - Consider a triple $\left(G, G^{\prime}, r\right)$ where $G$ is a group, $G^{\prime}$ is a subgroup of $G$, and $r$ is a retraction from $G$ onto $G^{\prime}$.

We say that $\left(G, G^{\prime}, r\right)$ is an extended hyperbolic floor if there exist a non trivial decomposition $\Gamma$ of $G$ as a graph of groups with surfaces, and a Bass-Serre presentation $\left(T^{1}, T^{0}\right)$ of $\Gamma$ such that:

- the surfaces of $\Gamma$ which are not once punctured tori have Euler characteristic at most -2;

- $G^{\prime}$ is the free product of the stabilizers of the non surface type vertices of $T^{0}$;

- every edge of $\Gamma$ joins a surface type vertex to a non surface type vertex (bipartism);

- either the retraction $r$ sends surface type vertex groups of $\Gamma$ to non abelian images; or $G^{\prime}$ is cyclic and there exists a retraction $r^{\prime}: G * \mathbb{Z} \rightarrow G^{\prime} * \mathbb{Z}$ which sends surface type vertex groups of $\Gamma$ to non abelian images.

We can then define extended hyperbolic towers from extended hyperbolic floors in exactly the same way that we defined hyperbolic towers from hyperbolic floors in Definition 5.4 of [1].

REMARK 3. - Note that all the extended hyperbolic floors $\left(G^{k}, G^{k+1}, r_{k}\right)$ of an extended hyperbolic tower must in fact be hyperbolic floors, except possibly for the bottom floor $\left(G^{m-1}, G^{m}, r_{m-1}\right)$. Moreover if this is the case, then $G^{m}$ must be cyclic, so $H$ is cyclic or trivial. In particular every extended hyperbolic tower structure over a non trivial non cyclic group is in fact a hyperbolic tower.

Note also that in parallel to Remark 5.5 of [1], the structure of extended hyperbolic tower is stable under free products and is transitive.

\section{Proving the main result}

We now show Theorem 1.2 can still be proved using Proposition 1 above instead of Proposition 5.11 of [1].

THEOREM 4 (Theorem 1.2 of [1]). - If $H$ is an elementary subgroup of a torsion-free hyperbolic group $G$, then $G$ admits a structure of hyperbolic tower over $H$.

Proof. - The case where $G$ is abelian is trivial, so we may assume that $G$, and thus $H$, is not abelian. The proof given in [1] proceeds by uncovering successive hyperbolic floors structures using Proposition 5.11, and combining them using stability under free products and transitivity of hyperbolic tower structure. Applying Proposition 1 instead will produce extended hyperbolic floors, which we noted we can also combine. We thus get that $G$ admits a structure of extended hyperbolic tower over $H$. However since $H$ is not abelian, Remark 3 implies that $G$ is in fact a hyperbolic tower over $H$.

$4^{\text {e }}$ SÉRIE - TOME $46-2013$ - No 5 


\section{Proof of Proposition 1}

The bulk of the proof of Proposition 5.11 in [1] consists in building hyperbolic floors from preretractions which satisfy either one of two sets of (weak) extra hypotheses: those of Lemma 7.25, or those of Lemma 7.26. The proof in these cases is not affected. However Proposition 7.28, which claims that the conclusions of Proposition 5.11 still hold in the special case when neither set of hypotheses is satisfied, is erroneous. Note that the corrections to be made thus affect Section 7.8 of [1] only.

Proposition 7.28 should be replaced by the following weaker statement

Proposition 5. - Let A be a torsion-free hyperbolic group which admits a cyclic JSJ-like decomposition $\Lambda$ which does not consist of a single surface type vertex. Let $f: A \rightarrow A$ be a non injective preretraction with respect to $\Lambda$.

Suppose that $\Lambda$ and $f$ satisfy neither the hypotheses of Lemma 7.25 of [1], nor those of Lemma 7.26 of [1]. Then there exists a retraction $r: A \rightarrow A^{\prime}$ to a proper subgroup of $A$ such that $\left(A, A^{\prime}, r\right)$ is an extended hyperbolic floor. If moreover $\Lambda$ has at least two surface type vertices, $\left(A, A^{\prime}, r\right)$ can be assumed to be a hyperbolic floor.

Recall that the hypotheses of Proposition 5 imply in particular that $\Lambda$ has a single non surface type vertex, whose corresponding group is a cyclic group $Z=\langle z\rangle$. Any boundary subgroup of a surface group of $\Lambda$ is thus generated by a conjugate of $z^{k}$ for some $k$, we call $k$ the boundary exponent corresponding to this boundary component. We first prove the following stronger version of Lemma 7.29.

Lemma 6. - Under the hypotheses of Proposition 5, there exists a surface $\Sigma$ of $\Lambda$ with boundary exponents $k_{1}, \ldots, k_{m}$ such that the sum $k_{1}+\cdots+k_{m}$ is even. If $\Sigma$ is in fact orientable, we have $k_{1}+\cdots+k_{m}=0$.

Moreover, if $\Sigma$ is (i) a 4-punctured sphere, (ii) a 3-punctured projective plane, (iii) a 2-punctured Klein bottle, we must have (up to reordering) in case (i) $k_{1}=-k_{2}$ and $k_{3}=-k_{4}$; in case (ii) $k_{1}=-k_{2}$ and $k_{3}$ even; in case (iii) $k_{1}=-k_{2}$, or $k_{1}$ and $k_{2}$ both even.

Proof. - Since the hypotheses of Lemma 7.26 of [1] are not satisfied, there exists a surface type vertex group $S$ of $\Lambda$ (with corresponding surface $\Sigma$ ) all of whose exterior surface type vertex group have abelian images by $f$. Lemma 7.29 of [1] shows that the first part of the statement holds for this choice of $\Sigma$. To prove the moreover part, note that $f(S)$ is not abelian, though $f(\hat{S})$ is for any exterior surface type vertex group $\hat{S}$ : the morphism $f$ must kill an element corresponding to a two-sided simple closed curve on $\Sigma$ which does not bound a Möbius band. Since $\Sigma$ is small, there are few possibilities and the result follows easily.

We then have the following analogue of Lemma 7.30.

Lemma 7. - Suppose $\Lambda$ admits a surface $\Sigma$ which satisfies the conclusion of Lemma 7.29. If $\Sigma$ has only one boundary component, then A admits a structure of extended hyperbolic floor, which can be assumed to be a hyperbolic floor if $\chi(\Sigma)<-2$ or if $\Lambda$ has surfaces other than $\Sigma$. 
Proof. - Note that $k_{1} \neq 0$, so $\Sigma$ is not orientable and $k_{1}$ is even, say $k_{1}=2 k$. If $S$ is the vertex group corresponding to $\Sigma$, the subgroup $S^{\prime}=\left\langle z^{k}, S\right\rangle_{A}$ is the fundamental group of the closed non orientable surface $\Sigma^{\prime}$ obtained by gluing a Möbius band $M$ along the boundary component of $\Sigma$. We have $S^{\prime}=\left\langle d_{1}, \ldots, d_{p} \mid d_{1}^{2} \ldots d_{p}^{2}=1\right\rangle$.

If $p>5$ there is a retraction $S^{\prime} \rightarrow\left\langle d_{1}, d_{2}\right\rangle_{S^{\prime}}$ given by $r\left(d_{3}\right)=d_{2}^{-1}, r\left(d_{4}\right)=d_{1}^{-1}$ and $r\left(d_{j}\right)=1$ for all $j>4$. It extends to a retraction which gives $(A, r(A), r)$ a structure of hyperbolic floor (with a single surface which is the once punctured connected sum of $p-2$ projective planes).

If $p=4$, let $s$ be the generator of an infinite cyclic group $\mathbb{Z}$. We define a retraction $r$ on $A * \mathbb{Z}$ by setting $r\left(d_{1}\right)=d_{1}, r\left(d_{2}\right)=d_{1}^{-1}$, and $r\left(d_{3}\right)=r\left(d_{4}^{-1}\right)=s$, and extending it to $A * \mathbb{Z}$ by the identity on the other surface type vertex groups of $\Lambda$ and on $\mathbb{Z}$. Thus $A$ has a structure of extended hyperbolic floor over a proper subgroup. If $\Lambda$ has surfaces other than $\Sigma$, we can further retract $A * \mathbb{Z}$ onto $\mathbb{Z}$ by sending $s$ to a non boundary element in one of the other surface groups: this gives a structure of hyperbolic floor.

The proof of Proposition 5 then goes as follows

Proof. - Let $\Sigma$ be the surface of $\Lambda$ which satisfies the conclusion of Lemma 7.29. By Lemma 7, we may assume $\Sigma$ has at least two boundary components.

If $\Sigma$ is an $m$-punctured surface of genus at least $1(m \geq 2)$, with the exception of a twice punctured torus, the retraction $r$ given in the proof of Proposition 7.28 does make $(A, r(A), r)$ into a hyperbolic floor. In the other cases, the surface retracted by $r$ is not complex enough to appear as the surface of a hyperbolic floor, or the image of the retraction is a cyclic subgroup (see Figures 1 and 2): this is where the mistake lies.
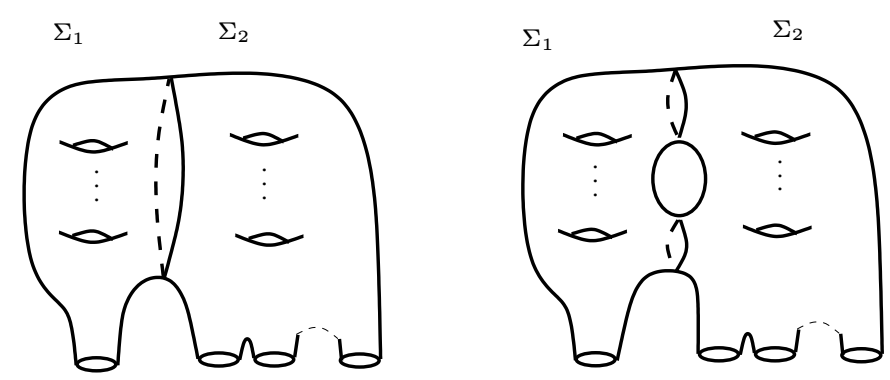

Figure 1. The proof of Proposition 7.28 proposes to retract the fundamental group of the surface $\Sigma_{2}$ on that of $\Sigma_{1}$ (in the even and odd genus cases respectively)...

We consider now the case of a twice punctured torus. The corresponding vertex group admits a presentation as $\left\langle x, y, b_{1}, b_{2} \mid[x, y]=b_{1} b_{2}\right\rangle$, and we must have $b_{1}=z^{k}$ and $b_{2}=t z^{-k} t^{-1}$ for $t$ a Bass-Serre element corresponding to the extra edge joining the surface vertex to the rigid vertex. The retraction which sends $x$ to $z^{k}, y$ to $t$, and is the identity on $Z$ gives a structure of hyperbolic floor for $A$ over the (free) subgroup generated by $z$ and $t$.

If $\Sigma$ has genus 0, it is an $m$-punctured (i) sphere, (ii) projective plane or (iii) Klein bottle. If $m$ is at least (i) 5 , (ii) 4 , and (iii) 3 respectively, then $A$ admits a structure of hyperbolic floor with one surface group corresponding to the complement of a thrice punctured sphere in $\Sigma$. 

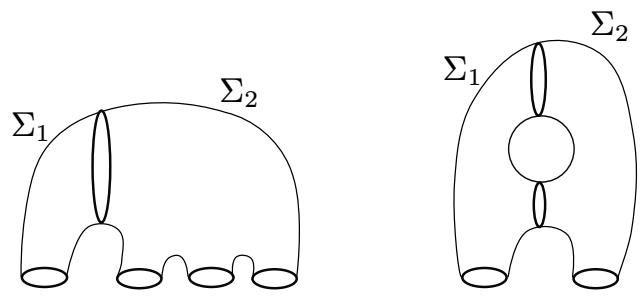

FIgURE 2. ... but in small complexity cases, the image of this retraction is cyclic (left) or the surface retracted is too small, so that this does not give a structure of hyperbolic floor.

Suppose now that $\Sigma$ is a (i) 4-punctured sphere, (ii) thrice punctured projective plane or (iii) twice punctured Klein bottle. By the moreover part of Lemma 6, we can choose

(i) $t, u, v$ elements of $A$ such that $z^{k_{1}} t z^{-k_{1}} t^{-1} u z^{k_{3}} u^{-1} v z^{-k_{3}} v^{-1}=1$;

(ii) $t, u, a$ elements of $A$ such that $z^{k_{1}} t z^{-k_{1}} t^{-1} u z^{2 \delta} u^{-1} a^{2}=1$;

(iii) $t, a, b$ elements of $A$ such that $z^{k_{1}} t z^{k_{2}} t^{-1} a^{2} b^{2}=1$ with $k_{1}=-k_{2}$ or $k_{1}=2 \delta$ and $k_{2}=2 \epsilon$.

We now define

(i) $r(t)=1$ and $r(u)=r(v)=s$;

(ii) $r(t)=1, r(u)=s$ and $r(a)=s z^{-\delta} s^{-1}$;

(iii) $r(t)=1, r(a)=s$ and $r(b)=s^{-1}$ if $k_{1}=-k_{2}$; and $r(t)=s, r(a)=s z^{-\epsilon} s^{-1}$ and $r(b)=z^{-\delta}$ otherwise;

where $s$ is a non trivial element a surface group of $\Lambda$ other than $S$ (if there are some), and the generator of an infinite cyclic group $\mathbb{Z}$ if not. We extend $r$ to $A$ (respectively to $A * \mathbb{Z}$ ) by the identity on the other surface type vertex groups of $A$ (respectively on $\mathbb{Z}$ ). This gives $A$ a structure of (extended) hyperbolic floor over $r(A)$ (respectively over $\langle z\rangle$ ).

REMARK 8. - The only cases in which the extended hyperbolic floor structures produced in Proposition 1 are not hyperbolic floors are thus when $\Lambda$ has a single non surface type vertex, which is cyclically stabilized, and a single surface vertex, which is stabilized by a surface group $S$ corresponding to (i) a once punctured connected sum of three projective plane; (ii) a twice punctured Klein bottle; (iii) a three punctured projective plane or (iv) a four punctured sphere.

\section{Towers in Sela's work}

Note that the definition of hyperbolic towers coincides exactly with that given in Sela's work, where they are called "non-elementary hyperbolic $\omega$-residually free towers" (see the paragraph before Proposition 6 in [4], and Definition 6.1 of [3] for the definition of $\omega$-residually free towers).

However, non-elementary hyperbolic $\omega$-residually free towers should also be replaced by extended hyperbolic towers for Proposition 6 of [4] to hold : a group $G$ that is elementary equivalent to a non abelian free group will only admit the structure of an extended hyperbolic 
tower over the trivial subgroup (recall that Proposition 5.11 of [1] is an intermediate step in the proof of Proposition 6 of [4], where it is not explicitly stated).

According to Sela, the converse of this result also holds, namely any extended hyperbolic tower over the trivial subgroup is elementary equivalent to the free group $\mathbb{F}_{2}$ [5].

\title{
REFERENCES
}

[1] C. Perin, Elementary embeddings in torsion-free hyperbolic groups, Ann. Sci. Éc. Norm. Supér. 44 (2011), 631-681.

[2] C. Perin, Elementary embeddings in torsion-free hyperbolic groups, updated version arXiv:0903.0945v3.

[3] Z. SEla, Diophantine geometry over groups. I. Makanin-Razborov diagrams, Publ. Math. I.H.É.S. 93 (2001), 31-105.

[4] Z. SELA, Diophantine geometry over groups. VI. The elementary theory of a free group, Geom. Funct. Anal. 16 (2006), 707-730.

[5] Z. SELA, private communication.

(Manuscrit reçu le 15 mai 2012;

accepté, après révision, le 4 juin 2013.)

\author{
Chloé Perin \\ Université de Strasbourg \\ I.R.M.A. \\ UMR CNRS 7501 \\ 7, rue René Descartes \\ 67084 Strasbourg Cedex, France \\ E-mail: cperin@math . unistra.fr
}

